\title{
Relativistic analysis of the LISA long range optical links
}

\author{
Bertrand Chauvineau, Tania Regimbau and Jean-Yves Vinet \\ Department ARTEMIS \\ Observatoire de la Côte d'Azur, \\ BP 42906304 Nice (France). \\ Sophie Pireaux \\ Department GEMINI \\ Observatoire de la Côte d'Azur, \\ Avenue Copernic 06130 Grasse (France).
}

(Dated: May 1, 2022)

\begin{abstract}
The joint ESA/NASA LISA mission consists in three spacecraft on heliocentric orbits, flying in a triangular formation of $5 \mathrm{Mkm}$ each side, linked by infrared optical beams. The aim of the mission is to detect gravitational waves in a low frequency band. For properly processing the scientific data, the propagation delays between spacecraft must be accurately known. We thus analyse the propagation of light between spacecraft in order to systematically derive the relativistic effects due to the static curvature of the Schwarzschild space-time in which the spacecraft are orbiting with time-varying light-distances. In particular, our analysis allows to evaluate rigorously the Sagnac effect, and the gravitational (Einstein) redshift.
\end{abstract}

PACS numbers: 04.80.Nn; 07.87.+v; 06.30.Ft; 04.25.-g; 04.25.Nx

\section{INTRODUCTION}

The three spacecraft forming the LISA constellation have a complex motion (see [1] for instance) allowing the triangular constellation to remain approximately rigid during the annual revolution. There is simultaneously a rotation of the triangle around its center, and the orbital motion of its center. A number of papers have considered the effect of the rotation on light propagation. Accurate knowledge of light propagation delays are very important for implementing the TDI (Time-Delay Interferometry) technique (see 2], 3]) which is mandatory for eliminating laser phase and similar noises. In particular, in a simulation code, it is necessary to generate time delays as realistic as possible. This is why we have carried out a full general relativistic treatment of light propagation in the gravitational potential of the Sun. The background metric created by the Sun is responsible for both the orbits, and the fine structure of time delays. Even if the orbit of any spacecraft is taken as purely Keplerian, the time interval between the emission of a photon off spacecraft A and its detection in spacecraft B must be thoroughly investigated. In particular, the motion of the target $\mathrm{B}$ during the interception must be evaluated with an accuracy level consistent with that of the null geodesic followed by the photon. We therefore propose an expansion of both the null geodesic equation from $\mathrm{A}$ to $\mathrm{B}$ and the motion of the target $\mathrm{B}$, in powers of the small dimensionless parameter

$$
\epsilon=r_{S} / 2 r=G M /\left(r c^{2}\right)
$$

where $r_{S}=2 G M / c^{2}$ is the Schwarzschild radius of the Sun (of mass $M$ ) and $r$ the coordinate distance of the spacecraft from the Sun. We intend to derive the time delay between spacecraft and the global frequency shift (including classical Doppler due to relative motion or orbital shift plus Einstein shift). The first quantity is essential for TDI, the second seems important for technology, because large frequency shifts, variable in time over a year induce requirements on the ultrastable oscillators used for compensation of orbital frequency shifts. We propose formulas for computing the time delays necessary for a realistic numerical model and we show that in the global frequency shift, the overall general relativistic contribution (first order terms) is much smaller than a naive estimate would suggest.

The method we present is based on a matching of geodesic equations of spacecrafts A and B, with the (null) geodesic equation of the photon emitted by $\mathrm{A}$ and received by B.

\section{COMPUTATION OF (COORDINATE-)TIME TRANSFER IN THE LITERATURE}

Different methods have been considered for computing the flight time of photons and the gravitational frequency shift between two space-time points $x_{A}^{\alpha} \equiv\left(t_{A}, \mathbf{x}_{A}\left(t_{A}\right)\right)$ (emitter) and $x_{B}^{\alpha} \equiv\left(t_{B}, \mathbf{x}_{B}\left(t_{B}\right)\right)$ (receiver). An alternative to integrating the geodesic equations of motion is to use the world function $\Omega\left(x_{A}^{\alpha}, x_{B}^{\alpha}\right)$ developed by Ruse [4] and Synge [5], defined as the squared geodesic distance between two space-time points with $\Omega\left(x_{A}^{\alpha}, x^{\alpha}\right)=0$ being the equation of the light cone at $x_{A}^{\alpha}$. The time delay corresponds to the future cone equation, and is given by $\tau\left(t_{A}, \mathbf{x}_{A}\left(t_{A}\right), \mathbf{x}_{B}\left(t_{B}\right)\right) \equiv t_{B}-t_{A}=R_{A B} / c-{ }_{\Omega}^{(P N)}$ $\left(t_{A}, \mathbf{x}_{A}\left(t_{A}\right), \mathbf{x}_{B}\left(t_{B}\right)\right) /\left(c R_{A B}\right)$, the subscript PN meaning Post-Newtonian contributions, $\mathbf{R}_{A B} \equiv \mathbf{x}_{B}\left(t_{B}\right)-$ $\mathbf{x}_{A}\left(t_{A}\right)$ and $R_{A B} \equiv\left|\mathbf{R}_{A B}\right|$.

Linet et al (2002) 6] provide the world function, 
$\Omega\left(t_{A}, \mathbf{x}_{A}\left(t_{A}\right), \mathbf{x}_{B}\left(t_{B}\right)\right)$, up to order $1 / c^{3}$ in the full Nordtvedt-Will Parametrized PN (PPN) formalism (10 parameters characterizing alternative theories of gravitation, including the usual $\mathrm{PN}$ parameters $\beta$ and $\gamma$ ). Their results yield an expression for the coordinate time transfer, $\tau\left(t_{A}, \mathbf{x}_{A}\left(t_{A}\right), \mathbf{x}_{B}\left(t_{\mathbf{B}}\right)\right)$, at order $1 / c^{4}$. The authors also provide $\tau\left(t_{A}, \mathbf{x}_{A}\left(t_{A}\right), \mathbf{x}_{B}\left(t_{\mathbf{A}}\right)\right)$ which contains explicitly the motion of the receiver and the corresponding Sagnac effects of orders $1 / c^{2}, 1 / c^{3}, 1 / c^{4}$. They applied the earlier expression to an isolated, axisymmetric rotating body, assuming a stationary gravitational field and a constant velocity of the central gravitational body with respect to the universe rest frame. Their systematic procedure to compute multipole moment contributions in $\Omega\left(\mathbf{x}_{A}\left(t_{A}\right), \mathbf{x}_{B}\left(t_{B}\right)\right)$ and $\tau\left(\mathbf{x}_{A}\left(t_{A}\right), \mathbf{x}_{B}\left(t_{B}\right)\right)$ was then particularized to obtain the explicit contributions of the mass monopole, mass quadrupole moment and of the intrinsic angular momentum of the rotating gravitational body. Restricting to fully conservative metric theories of gravitation, without preferred location effects (that is all PPN parameters vanish except $\beta$ and $\gamma$ ), they determined the fractional frequency shift up to order $1 / c^{4}$. The authors then performed numerical estimates of the frequency shifts in the gravitational field of the Earth. They assumed $A$ on board the International Space Station (ISS), orbiting at an altitude of $400 \mathrm{~km}$, and $B$ in a terrestrial station; as this is the case of ESA's Atomic Clock Ensemble in Space (ACES) mission, planned for 2009-2010, aiming at an accuracy of $10^{-16}$ in fractional frequency. The formula the authors give yields all the gravitational corrections to frequency shifts up to $10^{-18}$ in the vicinity of the Earth.

The above cited paper in fact generalizes the work of Blanchet et al (2001) 7], based on the geodesic approach, who provided the time transfer and frequency shifts up to order $1 / c^{3}$ in the setting of General Relativity (where $\beta=\gamma=1$ are the only nonvanishing PPN parameters), for a monopole mass, without intrinsic spin. Paper [7] considered the two-way laser link in addition to the oneway laser link presented in paper [6]. However, for the LISA mission, only single links are pertinent since laser light is not reflected from one station to another, LISA consisting in three pairs of laser links.

Le Poncin-Lafitte et al (2004) [8] present a generic procedure based on the world function to provide, in an iterative way, the propagation time of a photon between space-time events $x_{A}^{\alpha}$ and $x_{B}^{\alpha}$ at the $n$th postMinkowskian order (developpement in powers of $G$ ) from the contributions of orders $p=n-1, n-2, \ldots, 1,0$, i.e. of $\stackrel{(p)}{\Omega}\left(t_{A}, \mathbf{x}_{A}\left(t_{A}\right), \mathbf{x}_{B}\left(t_{B}\right)\right)$. The authors then apply their formalism to a static spherically symmetric spacetime within the second post-Minkowskian approximation, with the usual post-Newtonian parameters $\beta, \gamma$ plus parameter $\delta$ for the $G^{2}$-term in $g_{i j}$ (normalized so that in General Relativity $\delta=1$ ). Within those assumptions, they obtain the expressions of the world function and time transfer in the case of a simple monopolar and nonrotating body, up to order $G^{2}$ (containing order $1 / c^{5}$ ).
The motion of the stations (and corresponding Sagnac effects) is not explicitly considered there.

We are interested in the time variations of the interspacecraft propagation delays over a year for spacecraft having very special orbits, this is the reason why we develop a special approach, on principles analogous to the preceding works, but in a form more suitable to our purpose.

\section{POST-NEWTONIAN THEORY}

\section{A. Notations}

Coordinates $(c t, x, y, z)$ are denoted by $x^{\alpha}$. We use the notation $r=\sqrt{x^{i} x^{i}}$, where latin indices take only the spatial values 1,2 and 3 . Then, $r$ cannot be understood as a physical distance in all the steps of the developments. It should be referred to as a coordinate distance.

Four-velocities are written $u^{\alpha} \equiv d x^{\alpha} / d \tau$, where $\tau$ is the proper time, given by $c^{2} d \tau^{2}=-d s^{2}$, since the signature chosen for the metric is $(-,+,+,+)$. We frequently use the three-velocity $v^{i} \equiv d x^{i} / d t$. This quantity cannot be understood as a physical velocity in all the steps of the developments. When a quantity $Q$ is evaluated up to order $p$ (integer or half-integer) in $\epsilon$, it will be written

$$
\stackrel{(\rightarrow p)}{Q}=\stackrel{(0)}{Q}+\stackrel{(1 / 2)}{Q}+\ldots+\stackrel{(p)}{Q}
$$

(l)

where $Q$ is the contribution of order $l$.

The notation $\mathbf{U}$ represents the three numbers $U^{i}$, and U.V represents the sum $U^{i} V^{i} \equiv U^{1} U^{1}+U^{2} U^{2}+U^{3} U^{3}$. The notation $\mathbf{U}^{2}=\mathbf{U} . \mathbf{U}$ will also be used. $\eta_{\alpha \beta}=$ $(-1,+1,+1,+1)$ is the Minkowski metric.

In this paper, we derive the (relative) frequency shift of a photon, linking an emitter spacecraft $\mathrm{A}$ to a receiver spacecraft $\mathrm{B}$, up to order $3 / 2$ in $\epsilon$.

\section{B. Generalities on the metric}

We consider a metric of the generic static form

$$
d s^{2}=-I(\epsilon) c^{2} d t^{2}+J(\epsilon) \delta_{i j} d x^{i} d x^{j}
$$

where $I$ and $J$ are two functions depending on the theory (e.g. General Relativity, Scalar-Tensor theory,...). Both are assumed expansions in integer powers of $\epsilon$. The leading order in $v^{i} / c$, is $1 / 2$ (Kepler). A photon worldline, described by the wave vector $k^{\alpha} \equiv d x^{\alpha} / d \lambda$, where $\lambda$ is an (arbitrary) affine parameter, obeys the isotropic condition

$$
k_{\alpha} k^{\alpha}=0
$$

and the null geodesic equation

$$
\frac{d k^{\alpha}}{d \lambda}+\Gamma_{\mu \nu}^{\alpha} k^{\mu} k^{\nu}=0
$$


or, as well

$$
\frac{d k_{\alpha}}{d \lambda}=\frac{1}{2} k^{\mu} k^{\nu} \partial_{\alpha} g_{\mu \nu} .
$$

Since the considered metric is stationary, $k_{0} \equiv-I(\epsilon) k^{0}$ is a constant, according to (5).

A free falling observer of coordinates $x^{\alpha}$ and fourvelocity $u^{\alpha}$ can be described by the geodesic equation

$$
\frac{d u^{\alpha}}{d \tau}+\Gamma_{\mu \nu}^{\alpha} u^{\mu} u^{\nu}=0 .
$$

\section{Energy of a photon}

Consider a photon of four-wave vector $k^{\alpha}$ propagating in the background metric (2). The general expression of its energy measured by an observer of four-velocity $u^{\alpha}$ reads:

$$
E=-g_{\alpha \beta} k^{\alpha} u^{\beta}=-k_{0} u^{0}-J(\epsilon) \mathbf{k} . \mathbf{u} .
$$

An expansion of $E$ up to order $3 / 2$ requires a knowledge of $u^{0} / c$ and $u^{i} / c$ at the same level. On the other hand, $u^{i} / c$ being a term of order $1 / 2$ (i.e. $\left.\stackrel{(0)}{u}\right)^{i}=0$ ), we need $k^{i}$ and $J$ up to order 1 only, to evaluate $E$. Since the metric components are integer powers of $\epsilon$, the functions $I(\epsilon)$ and $J(\epsilon)$ have to be known up to order 1 only, in all the developments we will have to consider.

Let us compute $u^{0}=c . d t / d \tau$. We have

$$
c^{2} d \tau^{2}=I(\epsilon) c^{2} d t^{2}-J(\epsilon) d x^{i} d x^{i}=\left(I-J \frac{\mathbf{v}^{2}}{c^{2}}\right) c^{2} d t^{2} .
$$

Let us consider metrics of the form

$$
\begin{gathered}
I(\epsilon)=1-2 \epsilon+\mathcal{O}\left(\epsilon^{2}\right) \\
J(\epsilon)=1+2 \gamma \epsilon+\mathcal{O}\left(\epsilon^{2}\right)
\end{gathered}
$$

where we recognize the isotropic form of the Schwarzschild metric in the case where the PostNewtonian parameter $\gamma$ is unity. We thus have at the same order

$$
d \tau^{2}=\left(1-2 \epsilon-\frac{\mathbf{v}^{2}}{c^{2}}\right) d t^{2}
$$

and consequently

$$
\frac{u^{0}}{c}=1+\epsilon+\frac{\mathbf{v}^{2}}{2 c^{2}}+\mathcal{O}\left(\epsilon^{2}\right)
$$

We notice that proper and coordinate times differ by terms in $\epsilon$ (Einstein shift) and in $v^{2} / c^{2}$ (Lorentz time dilation). Now we compute $u^{i}$

$u^{i}=\frac{d x^{i}}{d \tau}=\frac{d t}{d \tau} \frac{d x^{i}}{d t}=\frac{u^{0}}{c} v^{i}=\left(1+\epsilon+\frac{\mathbf{v}^{2}}{2 c^{2}}\right) v^{i}+\mathcal{O}\left(\epsilon^{5 / 2}\right)$

from what we get the energy

$$
\frac{E}{c}=-k_{0}\left(1+\epsilon+\frac{\mathbf{v}^{2}}{2 c^{2}}\right)-\left[1+(1+2 \gamma) \epsilon+\frac{\mathbf{v}^{2}}{2 c^{2}}\right] \frac{\mathbf{k} \cdot \mathbf{v}}{c} .
$$

\section{Light propagation in curved space-time}

The metric tensor is of the form

$$
g_{\alpha \beta}=\eta_{\alpha \beta}+2 q_{\alpha} \epsilon \delta_{\alpha \beta}
$$

where $q_{0}=1$ and $q_{i}=\gamma$. We have as well

$$
g^{\alpha \beta}=\eta_{\alpha \beta}-2 q_{\alpha} \epsilon \delta^{\alpha \beta}
$$

The motion of the photon is described by the geodesic equation (4) or (5) and by the null condition (3).

\section{Order 0}

At this order, space-time is flat and the spacecrafts are at rest (no velocities, which are $1 / 2$ order terms, and no accelerations, which are first order terms), and light propagates according to special relativity. The results of order 0 are obvious, but since the same notation will be used in higher order terms, we present here the detailed solution.

Solving eq.(4), we find, the $\Gamma$ being first order terms

$$
\stackrel{(0)}{k}=C^{\alpha}
$$

where $C^{\alpha}$ are four integration constants. From the isotropic condition (3),

$$
C_{0} C_{0}=C^{0} C^{0}=C_{i} C_{i}=C^{i} C^{i}
$$

where $C_{\alpha} \equiv \eta_{\alpha \beta} C^{\beta}$, so that there is a spatial unit vector $n^{i} \equiv C^{i} / C^{0}$ along the direction of the (straight) light ray. We can parametrize the order 0 photon worldline using the trivial differential equation

$$
\frac{d \stackrel{(0)^{\alpha}}{d \lambda}}{d \lambda}=\stackrel{(0)}{k}
$$

which gives (assuming $\lambda=0$ for the emission time)

$$
\stackrel{(0)}{x}{ }^{0}=C^{0} \lambda
$$

and if $x_{\mathrm{A}}^{i}$ represents the coordinates of the emitter at emission time, we have

$$
\stackrel{(0)}{x}=x_{\mathrm{A}}^{i}+C^{i} \lambda
$$

Let us remark that interpreting $\stackrel{(0)}{x}^{0}$ as the Minkowski time coordinate $c t$, this could be simply written as

$$
\stackrel{(0)}{\mathbf{x}}(t)=\mathbf{x}_{\mathrm{A}}+c t . \mathbf{n} \text {. }
$$

The spatial coordinate distance $\stackrel{(0)}{r}(\lambda)$, which corresponds
to the "physical instantaneous distance" at this order, is:

$$
\stackrel{(0)}{r}(\lambda)^{2}=\stackrel{(0)}{x} \stackrel{i}{i} \stackrel{(0)}{x}=\left[C^{0} \lambda+\mathbf{n} \cdot \mathbf{x}_{\mathrm{A}}\right]^{2}+K^{2}
$$

with $K^{2}=r_{\mathrm{A}}^{2}-\left(\mathbf{n} \cdot \mathbf{x}_{\mathrm{A}}\right)^{2}$ and $r_{\mathrm{A}}^{2} \equiv \mathbf{x}_{\mathrm{A}}^{2}$. 


\section{Order 1}

$k_{0}$ being a constant, we obtain $k^{0}$ at order 1 by

$$
\stackrel{(\rightarrow 1)^{0}}{k}=g^{00} \stackrel{(\rightarrow 1)}{k}{ }_{0}=g^{00} \stackrel{(0)}{k}{ }_{0}=g^{00} C_{0}=(1+2 \stackrel{(0)}{\epsilon}) C^{0}
$$

where $\stackrel{(0)}{\epsilon}=2 r_{S} / \stackrel{(0)}{r}$. Integrating the spatial components of the geodesic equation (4), or (5), one finds

$$
\stackrel{(\rightarrow 1)^{i}}{k}=C^{0}\left[n^{i}-(1+\gamma) \stackrel{(0)}{\epsilon} P^{i} \mathbf{n} . \stackrel{(0)}{\mathbf{x}}-(\gamma-1) \stackrel{(0)}{\epsilon} n^{i}\right]
$$

where $P^{i}$ is defined as

$$
P^{i} \equiv \frac{x_{\mathrm{A}}^{i}-n^{i} \mathbf{n} \cdot \mathbf{x}_{\mathrm{A}}}{K^{2}}
$$

and satisfies $\mathbf{P . n}=0$. Integrating equation

$$
\frac{d \stackrel{(\rightarrow 1)^{\alpha}}{x}}{d \lambda}=\stackrel{(\rightarrow 1)^{\alpha}}{k}
$$

one finds, in parametric form

$$
\begin{aligned}
& \stackrel{(\rightarrow 1)}{x}^{0}(\lambda)=C^{0} \lambda+2 \frac{G M}{c^{2}} \ln \frac{\mathbf{n} \cdot \stackrel{(0)}{\mathbf{x}}+\stackrel{(0)}{r}}{\mathbf{n} \cdot \mathbf{x}_{\mathrm{A}}+r_{\mathrm{A}}} \\
& \stackrel{(\rightarrow 1)^{i}}{x}(\lambda)=x_{\mathrm{A}}^{i}+C^{i} \lambda-(\gamma+1) \frac{G M}{c^{2}} P^{i}\left[\stackrel{(0)}{r}-r_{\mathrm{A}}\right] \\
& -(\gamma-1) \frac{G M}{c^{2}} n^{i} \ln \frac{\mathbf{n} \cdot \stackrel{(0)}{\mathbf{x}}+\stackrel{(0)}{r}}{\mathbf{n} \cdot \mathbf{x}_{\mathrm{A}}+r_{\mathrm{A}}} .
\end{aligned}
$$

Eliminating the affine parameter, one obtains the photon's trajectory in the explicit form

$$
\stackrel{(\rightarrow 1)}{x}^{i}\left(t, n^{j}\right)=x_{\mathrm{A}}^{i}+n^{i} c t-(\gamma+1) \frac{G M}{c^{2}} \chi^{i}\left(t, n^{j}\right)
$$

with

$$
\chi^{i}\left(t, n^{j}\right) \equiv P^{i}\left[\stackrel{(0)}{r}-r_{\mathrm{A}}\right]+n^{i} \ln \frac{\mathbf{n} \cdot \stackrel{(0)}{\mathbf{x}}+\stackrel{(0)}{r}}{\mathbf{n} \cdot \mathbf{x}_{\mathrm{A}}+r_{\mathrm{A}}}
$$

where the dependence in the integrations constants $n^{i}$ is explicitely written and $\stackrel{(0)}{r}$ is given by (11) with $C^{0} \lambda$ replaced by $c t$.

\section{Energy of the photon}

Inserting (13) in (8), one finds

$$
\frac{1}{C^{0}} \frac{E}{c}=1-\frac{\mathbf{n} \cdot \mathbf{v}}{c}+\frac{G M}{r c^{2}}+\frac{\mathbf{v}^{2}}{2 c^{2}}
$$

$$
\begin{gathered}
-\left[(2+\gamma) \frac{G M}{\stackrel{(0)}{r} c^{2}}+\frac{\mathbf{v}^{2}}{2 c^{2}}\right] \frac{\mathbf{n} \cdot \mathbf{v}}{c} \\
+(\gamma+1) \frac{G M}{c^{2}} \frac{\mathbf{P} \cdot \mathbf{v}}{c} \frac{\mathbf{n} \cdot \stackrel{(0)}{\mathbf{x}}}{\stackrel{(0)}{r}}
\end{gathered}
$$

The first line of the right-hand side (r.h.s.) of (15) contains terms of order 0 (i.e. 1), 1/2 (i.e. $-\mathbf{n . v} / c$ ) and 1 , while terms of order $3 / 2$ are in the second and third lines.

Equation (15) shows that knowledge of the energy up to order $3 / 2$ requires

- knowledge of $n^{i}$ up to order 1 ;

- knowledge of $v^{i} / c$ up to order $3 / 2$;

- knowledge of $x^{i}$ up to order $1 / 2$.

\section{Evaluating the flight time and the constants $n^{i}$}

At $t=0$, a photon is emitted from $x_{\mathrm{A}}^{i}$ by spacecraft $\mathrm{A}$ with initial position $x_{\mathrm{A}}^{i}$ and velocity $v_{\mathrm{A}}^{i}$, and is received at $x_{\mathrm{B}}^{i}(t)$ by spacecraft $\mathrm{B}$ with initial position $x_{\mathrm{B}}^{i}$ and velocity $v_{\mathrm{B}}^{i}$. We want to compute both the flight time $t$, and the constants $n^{i}$ characterizing this photon.

The equation to solve writes

$$
x^{i}\left(t, n^{j}\right)=x_{\mathrm{B}}^{i}(t)
$$

where $x^{i}\left(t, n^{j}\right)$ represents the photon's trajectory (14) and $x_{\mathrm{B}}^{i}(t)$ the receiver spacecraft's trajectory. Since $i=$ $1,2,3$, equation (16) represents three equations involving the four unknown $t$ and $n^{i}$. The fourth equation is the normalization condition

$$
\mathbf{n}^{2}=n^{i} n^{i}=1
$$

The Taylor development reads

$$
x_{\mathrm{B}}^{i}(t)=x_{\mathrm{B}}^{i}+t v_{\mathrm{B}}^{i}+\frac{t^{2}}{2}\left[\frac{d v_{\mathrm{B}}^{i}}{d t}\right]_{(t=0)}+\ldots
$$

Since the operator $d / d t=v^{i} \partial_{i}$ increases by $1 / 2$ the order of the quantity on which it is applied, this development shows that:

- knowledge of $x_{\mathrm{B}}^{i}(t)$ up to $1 / 2$ order requires knowledge of $t$ at order 0 ;

- knowledge of $v_{\mathrm{B}}^{i}(t)=d x_{\mathrm{B}}^{i}(t) / d t$ up to order $3 / 2$ requires knowledge of $t$ at order $1 / 2$.

Hence, one has to solve equation (16) up to order $1 / 2$ for $t$ and up to order 1 for $n^{i}$.

Let us write (16) in the explicit form, using (14) and (18),

$x_{\mathrm{A}}^{i}+n^{i} c t-(\gamma+1) \frac{G M}{c^{2}} \chi^{i}\left(t, n^{j}\right)=x_{\mathrm{B}}^{i}+t v_{\mathrm{B}}^{i}-\frac{t^{2}}{2} \frac{G M x_{\mathrm{B}}^{i}}{r_{\mathrm{B}}^{3}}$

where the initial acceleration, given by the geodesic equation (66) reduces to its newtonian part at order 1 . We 
notice that, due to properties of the metric considered (static and diagonal), this is also the expression of the initial acceleration up to order $3 / 2$.

At order 0, equations (19) and (17) read

$$
\begin{gathered}
x_{\mathrm{A}}^{i}+\stackrel{(0)}{n}^{i} c \stackrel{(0)}{t}=x_{\mathrm{B}}^{i} \\
\stackrel{(0)^{2}}{\mathbf{n}}=1
\end{gathered}
$$

which give the two (obvious) order 0 solutions

$$
\begin{aligned}
& c \stackrel{(0)}{t}= \pm \sqrt{\left(x_{\mathrm{B}}^{i}-x_{\mathrm{A}}^{i}\right)\left(x_{\mathrm{B}}^{i}-x_{\mathrm{A}}^{i}\right)} \\
& \stackrel{(0)}{n}= \pm \frac{x_{\mathrm{B}}^{i}-x_{\mathrm{A}}^{i}}{\sqrt{\left(x_{\mathrm{B}}^{j}-x_{\mathrm{A}}^{j}\right)\left(x_{\mathrm{B}}^{j}-x_{\mathrm{A}}^{j}\right)}}
\end{aligned}
$$

where the "+" sign corresponds to a photon emitted by spacecraft A and received by spacecraft B, while the "-" sign corresponds to a photon emitted by spacecraft B and received by spacecraft $\mathrm{A}$. This is nothing but the trivial euclidean prediction in the case where both emitting and receiving spacecrafts are at rest.

At order $1 / 2$, using the 0 order equations, equations (19) and (17) read

$$
\begin{gathered}
\stackrel{(0)}{n})^{i}(1 / 2) \\
t \\
+\stackrel{(0)}{t} \stackrel{(1 / 2)^{i}}{n}=\stackrel{(0)}{t} \frac{v_{\mathrm{B}}^{i}}{c} \\
\stackrel{(0)}{\mathbf{n}} . \stackrel{(1 / 2)}{\mathbf{n}}=0 .
\end{gathered}
$$

The corresponding terms of order $1 / 2$

$$
\begin{gathered}
\stackrel{(1 / 2)}{t}=\stackrel{(0)}{t} \stackrel{\stackrel{(0)}{\mathbf{n}} \cdot \mathbf{v}_{\mathrm{B}}}{c}=\frac{v_{\mathrm{B}}^{i}}{c^{2}}\left[x_{\mathrm{B}}^{i}-x_{\mathrm{A}}^{i}\right] \\
\stackrel{(1 / 2)^{i}}{=}=\frac{v_{\mathrm{B}}^{i}}{c}-\stackrel{(0)^{i}}{n} \frac{\stackrel{(0)}{\mathbf{n}} \cdot \mathbf{v}_{\mathrm{B}}}{c}
\end{gathered}
$$

are the classical motion and aberration corrections.

The same iterative method provides the next order terms. For the energy problem, one only needs $\stackrel{(1)}{n}^{i}$, but the method also gives $\stackrel{(1)}{t}$, which will be useful in the time-delay problem

$$
\begin{gathered}
\stackrel{(1)}{t}=\frac{1}{2}\left[\frac{\mathbf{v}_{\mathrm{B}}^{2}}{c^{2}}+\left(\frac{\stackrel{(0)}{\mathbf{n}} \cdot \mathbf{v}_{\mathrm{B}}}{c}\right)^{2}\right] \stackrel{(0)}{t}+ \\
\left.+\stackrel{(0)}{n})^{i}\left[(1+\gamma) \frac{G M}{c^{3}} \chi^{i} \stackrel{(\stackrel{0}{t}, \stackrel{(0)}{n})^{j}}{\mathrm{t}}\right)-\frac{G M x_{\mathrm{B}}^{i}}{2 r_{\mathrm{B}}^{3} c} \stackrel{(0)^{2}}{t}\right]
\end{gathered}
$$

$$
\begin{gathered}
\stackrel{(1)}{n}^{i}=-\frac{1}{2 c^{2}} \stackrel{(0)^{i}}{n}\left[\mathbf{v}_{\mathrm{B}}^{2}-\left(\stackrel{(0)}{\mathbf{n}} \cdot \mathbf{v}_{\mathrm{B}}\right)^{2}\right] \\
-\frac{G M \stackrel{(0)}{t}}{2 r_{\mathrm{B}}^{3} c}\left[x_{\mathrm{B}}^{i}-\stackrel{(0)^{i}}{n} \stackrel{(0)}{\mathbf{n}} \cdot \mathbf{x}_{\mathrm{B}}\right] \\
+(\gamma+1) \frac{G M}{c^{3} \stackrel{(0)}{t}}\left[\chi^{i}-\stackrel{(0)^{i}}{n} \stackrel{(0)}{\mathbf{n}} \cdot \chi\right] \underset{\left.\left(\begin{array}{c}
(0) \\
t,{ }^{(0)}
\end{array}\right)^{k}\right)}{ } .
\end{gathered}
$$

The "ln" term in $\stackrel{(1)}{t}$, present in the $\chi^{i}$ term, is responsible for the well-known Shapiro time-delay effect.

\section{TIME DELAYS}

The time transfer between two satellites A and B is, at order 0 ,

$$
\stackrel{(\rightarrow 1)}{t}=\stackrel{(0)}{t}+\stackrel{(1 / 2)}{t}+\stackrel{(1)}{t}
$$

where the contributions $\stackrel{(k)}{t}$ have been calculated in the previous section. The 0 order term is the same for both links $\mathrm{A} \rightarrow \mathrm{B}$ and $\mathrm{B} \rightarrow \mathrm{A}$. The first term to be affected by the permutation $\mathrm{A} \rightleftharpoons \mathrm{B}$ is the $1 / 2$ order term. It is easy to see that, for this permutation, one has

$$
(\stackrel{(0)}{t}, \stackrel{(0)}{n})
$$

leading to

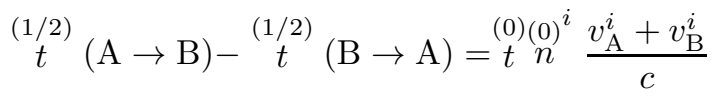

which is responsible for the so-called Sagnac effect. Let us notice that in the TDI problem, one generally needs the emission time as a function of the reception time (or, equivalently, the flight time as a function of the reception time), rather than the converse. Accordingly, the solution required in simulations generally involves the order 0 solution (20), (21) with the minus sign.

\section{EINSTEIN FREQUENCY SHIFTS}

Three identical clocks, one aboard each LISA spacecraft, beat at a common proper frequency. The quantity measured on spacecraft B is the (relative) difference between the frequency received from spacecraft $\mathrm{A}$, and the proper frequency measured on B. For a photon starting from $\mathrm{A}$ at $t=0$, and received at $\mathrm{B}$ at time $t$, this relative frequency shift writes

$$
z_{(\mathrm{B} \leftarrow \mathrm{A})}\left(t_{\mathrm{A}}=0\right)=\frac{E_{\mathrm{B}}(t)}{E_{\mathrm{A}}(t=0)}-1
$$


since the nominal frequency of the oscillator aboard $B$ is identical to the one of the oscillator aboard A, which is nothing but the initial energy of the photon emitted from A.

Let us remark that $1+z$ as defined in (24) corresponds to the standard definition of a frequency shift (frequency at reception over frequency at emission) in the case where the solution (2021) with the + sign is considered. In the other case ( $-\operatorname{sign}), 1+z$ as defined in (24) corresponds to the inverse ratio (frequency at emission over frequency at reception).

\section{A. Contribution of the different orders}

The development of the expression for the energy up to order $3 / 2$ is given by (15). This expression requires the velocity of the spacecraft up to order $3 / 2$

$$
\begin{gathered}
v_{\mathrm{B}}^{(3 / 2)^{i}}(t)=v_{\mathrm{B}}^{i}+t \frac{d v_{\mathrm{B}}^{i}}{d t}+\frac{t^{2}}{2} \frac{d^{2} v_{\mathrm{B}}^{i}}{d t^{2}} \\
=v_{\mathrm{B}}^{i}-t G M \frac{x_{\mathrm{B}}^{i}}{r_{\mathrm{B}}^{3}}-\frac{t^{2}}{2} G M\left[\frac{v_{\mathrm{B}}^{i}}{r_{\mathrm{B}}^{3}}-3 \frac{x_{\mathrm{B}}^{i} \mathbf{x}_{\mathrm{B}} \cdot \mathbf{v}_{\mathrm{B}}}{r_{\mathrm{B}}^{5}}\right] .
\end{gathered}
$$

Substituting the above expression in equation (15), one finds, writing $\Delta E \equiv E_{\mathrm{B}}(t)-E_{\mathrm{A}}(t=0)$

$$
\Delta E=\stackrel{(1 / 2)}{\Delta} E+\stackrel{(1)}{\Delta E}+\stackrel{(3 / 2)}{\Delta E}
$$

with, writing $N \equiv \Delta E /\left(c C^{0}\right)$,

$$
\begin{aligned}
& \stackrel{(1 / 2)}{N}=-\stackrel{(0)}{n} \frac{i}{v_{\mathrm{B}}^{i}-v_{\mathrm{A}}^{i}} \\
& \stackrel{(1)}{N}=\frac{v_{\mathrm{B}}^{i}-v_{\mathrm{A}}^{i}}{c^{2}}\left[\stackrel{(0)}{n} \stackrel{i}{n} \stackrel{(0)}{\mathbf{n}} \cdot \mathbf{v}_{\mathrm{B}}-\frac{1}{2}\left(v_{\mathrm{B}}^{i}-v_{\mathrm{A}}^{i}\right)\right] \\
& +\frac{G M}{c^{2}}\left[\frac{1}{r_{\mathrm{B}}}-\frac{1}{r_{\mathrm{A}}}\right]+\frac{G M}{c} \stackrel{(0)}{t} \frac{\stackrel{(0)}{\mathbf{n}} \cdot \mathbf{x}_{\mathrm{B}}}{r_{\mathrm{B}}^{3}}
\end{aligned}
$$

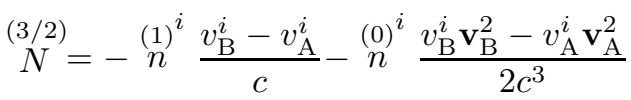

$$
\begin{aligned}
& -\frac{G M}{c^{2}} \stackrel{(0)}{t} \frac{\mathbf{v}_{\mathrm{B}} \cdot \mathbf{x}_{\mathrm{B}}}{r_{\mathrm{B}}^{3}}+\frac{G M}{2 c} \stackrel{(0)^{i}}{n} \stackrel{(0)^{2}}{t}\left[\frac{v_{\mathrm{B}}^{i}}{r_{\mathrm{B}}^{3}}-3 \frac{x_{\mathrm{B}}^{i} \mathbf{x}_{\mathrm{B}} \cdot \mathbf{v}_{\mathrm{B}}}{r_{\mathrm{B}}^{5}}\right] \\
& -(2+\gamma) \frac{G M}{c^{3}} \stackrel{(0)}{n}{ }^{i}\left[\frac{v_{\mathrm{B}}^{i}}{r_{\mathrm{B}}}-\frac{v_{\mathrm{A}}^{i}}{r_{\mathrm{A}}}\right] \\
& \left.+(1+\gamma) \frac{G M}{c^{3}} \stackrel{(0)^{i}}{P} \stackrel{(0)}{n}\right)^{j}\left[\frac{x_{\mathrm{B}}^{j} v_{\mathrm{B}}^{i}}{r_{\mathrm{B}}}-\frac{x_{\mathrm{A}}^{j} v_{\mathrm{A}}^{i}}{r_{\mathrm{A}}}\right]
\end{aligned}
$$

he first term originates in the Einstein gravitational Doppler effect, while the second is related to the accelerations of spacecrafts in the gravitational field of the Sun.

\section{B. Orders of magnitude for the LISA configuration}

One is tempted to derive an order of magnitude for the contributions to the frequency shift. Indeed, one has, from (11)

$$
\epsilon \sim 10^{-8}
$$

and the difference between the distance of each station to the Sun satisfies

$$
\frac{\delta r}{r} \leq \frac{L}{r} \cos \frac{\pi}{3} \sim \frac{1}{60}
$$


where $L \sim 5.10^{6} \mathrm{~km}$ is the typical interspacecraft distance. An estimation of the terms of orders $1 / 2,1$ and $3 / 2$ leads to

$$
\begin{gathered}
\stackrel{(1 / 2)}{z} \sim \delta\left(\epsilon^{1 / 2}\right) \sim \epsilon^{1 / 2} \frac{\delta r}{r} \sim 2.10^{-6} \\
\stackrel{(1)}{z} \sim \delta(\epsilon) \sim \epsilon \frac{\delta r}{r} \sim 2.10^{-10} \\
\stackrel{(3 / 2)}{z} \sim \delta\left(\epsilon^{3 / 2}\right) \sim \epsilon^{3 / 2} \frac{\delta r}{r} \sim 2.10^{-14} .
\end{gathered}
$$

It is interesting to point out that, due to the peculiar LISA configuration, terms of orders $1 / 2$ and 1 are actually considerably smaller than the above estimates. Let us evaluate these terms more carefully.

In the ideal case, where LISA spacecraft form a perfect equilateral triangle, velocities of spacecrafts $\mathrm{A}$ and $\mathrm{B}$ have the same projection on vector $\stackrel{(0)}{\mathbf{n}}$, such that from (29),

$$
\stackrel{(1 / 2)}{z}=0 .
$$

In fact, the above cancellation is obtained as soon as the orbits about the center of mass of the satellite constellation are coplanar and circular, with a velocity proportional to the center of mass distance. Since this property is not exactly verified by the real configuration, the value of $\stackrel{(1 / 2)}{z}$ is reduced by a factor $\sim L / r$, i.e. $\stackrel{(1 / 2)}{z} \sim 7 \cdot 10^{-8}$. At this order, the frequency shift is due to the arm length variations (flexing).

Let $\mathbf{v}_{\mathrm{AB}} \equiv \mathbf{v}_{\mathrm{B}}-\mathbf{v}_{\mathrm{A}}$. From (30), (27), (26) and (28), the term of order 1 writes

$$
\begin{gathered}
\stackrel{(1)}{z}=\left(\frac{(0)}{\stackrel{\mathbf{n}}{\mathbf{v}_{\mathrm{AB}}}}\right)^{2}-\frac{1}{2}\left(\frac{\mathbf{v}_{\mathrm{AB}}}{c}\right)^{2} \\
+\frac{G M}{c^{2}}\left(\frac{1}{r_{\mathrm{B}}}-\frac{1}{r_{\mathrm{A}}}\right)+\frac{G M}{c} \stackrel{(0)}{t} \frac{\stackrel{(0)}{\mathbf{n}} \cdot \mathbf{x}_{\mathrm{B}}}{r_{\mathrm{B}}^{3}} .
\end{gathered}
$$

The first term on the rhs is small, $\sim 5 \cdot 10^{-15}$, since it is nothing but $\stackrel{(1 / 2)^{2}}{ }$.

In the second term, $\mathbf{v}_{\mathrm{AB}}^{2}$ turns out to be three times the squared modulus of the relative velocity of a spacecraft with respect to LISA's center of mass, i.e. $V L /(r \sqrt{3})$ with $V$ the orbital velocity of this center. Hence,

$$
\frac{1}{2}\left(\frac{\mathbf{v}_{\mathrm{AB}}}{c}\right)^{2} \sim \frac{V^{2}}{c^{2}} \frac{L^{2}}{2 r^{2}} \sim 6.10^{-12} .
$$

The third term of the rhs of (31) is of order

$$
\frac{G M}{r c^{2}} \frac{\delta r}{r} \sim 2.10^{-10}
$$

while the fourth writes

$$
\frac{G M}{c^{2}} c \stackrel{(0)}{t} \frac{\stackrel{(0)}{\mathbf{n}} \cdot \mathbf{x}_{\mathrm{B}}}{r_{\mathrm{B}}^{3}} \sim \frac{G M}{r c^{2}} \frac{L}{r} \cos \theta \sim 2.10^{-10}
$$

where $\theta$ spans between $\pi / 3$ and $2 \pi / 3$.

The point is that the third and fourth rhs terms of equation (31), of order $\left(G M / c^{2}\right)\left(L / r^{2}\right)$ each, nearly cancel. Indeed, the euclidean triangular assumption,

$$
r_{\mathrm{A}}^{2}=r_{\mathrm{B}}^{2}-2 r_{\mathrm{B}} \mathbf{L} \cdot \widehat{\mathbf{x}}_{\mathrm{B}}+L^{2}
$$

with $\mathbf{x}_{\mathrm{A}, \mathrm{B}}=r_{\mathrm{A}, \mathrm{B}} \widehat{\mathbf{x}}_{\mathrm{A}, \mathrm{B}}$ and $\mathbf{L}=c \stackrel{(0)(0)}{t} \stackrel{\mathbf{n}}{\text { leads to }}$

$\frac{1}{r_{\mathrm{A}}}=\frac{1}{r_{\mathrm{B}}}+\frac{\mathbf{L} \cdot \widehat{\mathbf{x}}_{\mathrm{B}}}{r_{\mathrm{B}}^{2}}+\left[-1+3\left(\stackrel{(0)}{\mathbf{n}} \cdot \widehat{\mathbf{x}}_{\mathrm{B}}\right)^{2}\right] \frac{L^{2}}{2 r_{\mathrm{B}}^{3}}+\mathcal{O}\left(\frac{L^{3}}{r^{4}}\right)$.

Since $\left(\stackrel{(0)}{\mathbf{n}} \cdot \widehat{\mathbf{x}}_{\mathrm{B}}\right)^{2} \leq 1 / 4$, the sum of the third and fourth terms in (31) is about

$$
\frac{1}{2} \frac{G M}{r c^{2}}\left(\frac{L}{r}\right)^{2} \sim 6.10^{-12} .
$$

Furthermore, it turns out that the residual (third+fourth)-term in (31), of order $\left(G M / c^{2}\right)\left(L^{2} / r^{3}\right)$, nearly cancels with the second term in (31). Indeed, at first order, each spacecraft obeys Newton's law

$$
\frac{d \mathbf{v}}{d t}=-G M \frac{\mathbf{x}}{r^{3}}
$$

so that their relative motion is described by

$$
\frac{d \mathbf{v}_{\mathrm{AB}}}{d t}=-G M\left(\frac{\mathbf{L}}{r^{3}}-3 \frac{(\mathbf{r} . \mathbf{L}) \mathbf{r}}{r^{5}}\right)+\mathcal{O}\left(\frac{L^{2}}{r^{4}}\right)
$$

with $\mathbf{v}_{\mathrm{AB}}=d \mathbf{L} / d t$. Since $d\left(\mathbf{L} \cdot \mathbf{v}_{\mathrm{AB}}\right)=\mathbf{L} \cdot d \mathbf{v}_{\mathrm{AB}}+\mathbf{v}_{\mathrm{AB}}^{2} d t$, it turns out that the sum of the second, third and fourth terms of (31) reduces to

$$
-\frac{1}{2 c^{2}} \frac{d}{d t}\left(L \stackrel{(0)}{\mathbf{n}} \cdot \mathbf{v}_{\mathrm{AB}}\right)+\mathcal{O}\left(\frac{L^{3}}{r^{4}}\right) .
$$

The order of magnitude of the $\mathcal{O}\left(L^{3} / r^{4}\right)$ terms neglected at the different steps leading to (34) is

$$
\frac{G M}{c^{2}} \frac{L^{3}}{r^{4}} \cos \theta \sim 2.10^{-13} .
$$

But the first term of (34) is even smaller, since

$$
\frac{1}{2 c^{2}} \frac{d}{d t}\left(L \stackrel{(0)}{\mathbf{n}} \cdot \mathbf{v}_{\mathrm{AB}}\right) \sim \frac{1}{2 c} L \frac{(1 / 2)}{1 \mathrm{yr}} \sim 3 \cdot 10^{-14} .
$$

Consequently, $\stackrel{(1)}{z}$ appears to be of order $2.10^{-13}$, i.e. only an order of magnitude larger than $\underset{z}{(3 / 2)}$.

In the next section, we will confirm the partial cancelling of $\stackrel{(1 / 2)}{z}$ and $\stackrel{(1)}{z}$ found analytically, thanks to a numerical relativistic simulation of LISA's optical links. 


\section{NUMERICAL RESULTS}

In order to evaluate the orders of magnitude of the various terms of the expansions in the time delays and in the frequency shifts, we have made a toy model in which the spacecraft orbits are classical (Keplerian). At any date, the link between spacecraft $\mathrm{A}$ and $\mathrm{B}$ is assumed starting from the Keplerian location of A at current time $t_{A}$ and joining $\mathrm{B}$ at a place deduced from the keplerian position of $\mathrm{B}$ at time $t_{A}+t$, through a relativistic continuation. It is known (this has been discussed for instance recently in [1]), that the three spacecraft can be kept at approximately constant mutual distances, with the constellation's center of mass on a circular orbit of 1 A.U. radius, at the condition that each orbit has a small eccentricity $e$, a small orbital inclination $\mu$ on the ecliptic, with the semimajor axes of spacecraft 2 and spacecraft 3 orbits shifted by 120 and 240 degrees respectively from spacecraft 1 's. In fact, if the three spacecraft are required to be equivalent (i.e., same orbit eccentricity $e$ and inclination angle $\mu$ ), at first order in $e$, the distances between spacecraft are constant and the angle of the LISA plane with respect to the ecliptic, $\nu$, is 60 degrees. However, exact numerical investigations show that second order terms in $e$ give rise to important variations of the interferometric arm lenghts ("breathing") and to a large Doppler effect. The choice of the orbital parameters $(e, \nu)$ influences the "breathing" amplitude. A carefull choice of the orbital parameters can reduce the "breathing" amplitude from about $120,000 \mathrm{~km}$ (for a naive choice of $\nu=60$ degrees) to less than $50,000 \mathrm{~km}$. Such orbital parameters were chosen in existing simulation codes [9], 10], and it has been shown in reference [11] that this choice is the optimal one. We summarize herafter the results of [11] in terms of parametrized orbits. The angle $\nu$ is defined as

$$
\nu=\frac{\pi}{3}+\frac{5}{8} \alpha
$$

where $\alpha \equiv L / 2 R$. $L$ is the nominal interspacecraft distance $\left(510^{6} \mathrm{~km}\right)$ and $R$ the radius of the circular LISA orbit ( 1 a.u.), so that $\alpha \sim 1 / 60$ is a small parameter. This small change of inclination is responsible for a large reduction of flexing with respect to the strict 60 degrees often quoted in the literature 1]. The inclination $\mu$ of the orbital planes with respect to the ecliptic is:

$$
\tan \mu=\frac{\alpha \sin \nu}{\sin (\pi / 3)+\alpha \cos \nu}
$$

Though the center of mass keeps on a circular orbit, the spacecraft stay on slightly elliptical orbits (numbered $b=$ $1,2,3)$. Their common eccentricity is:

$$
e=\left[1+\frac{4 \alpha \cos \nu}{\sqrt{3}}+\frac{4 \alpha^{2}}{3}\right]^{1 / 2}-1
$$

In order to parametrize the orbital motions by the time, we need the eccentric anomalies $\Psi_{b}$, that are functions of the time $t$, defined via the implicit equations:

$$
\Psi_{b}-e \sin \Psi_{b}=\Omega t-\theta_{b}
$$

involving a phase shift $\theta_{b} \equiv 2(b-1) \pi / 3$. Owing to the smallness of $e$, the numerical solution is very fast. Then, let us set

$$
\left\{\begin{array}{l}
X_{b}=R\left(\cos \Psi_{b}-e\right) \cos \mu \\
Y_{b}=R \sqrt{1-e^{2}} \sin \Psi_{b} \\
Z_{b}=-R\left(\cos \Psi_{b}-e\right) \sin \mu
\end{array}\right.
$$

Finally, the positions of the three spacecraft at time $t$ are deduced from the preceding ones via rotations in the $(X, Y)$-plane:

$$
\left\{\begin{array}{l}
x_{b}=X_{b} \cos \theta_{b}-Y_{b} \sin \theta_{b} \\
y_{b}=X_{b} \sin \theta_{b}+Y_{b} \cos \theta_{b} \\
z_{b}=Z_{b}
\end{array}\right.
$$

At this point, we have three Keplerian orbits, with parameters such that the distances between test masses are constant at first order in $\alpha$, but actually varying by about $1 \%$ (about $50,000 \mathrm{~km}$ ) peak-to-peak during the year. The spacecraft velocities are computed according to:

$$
\left\{\begin{array}{l}
\dot{x}_{b}=\dot{X}_{b} \cos \theta_{b}-\dot{Y}_{b} \sin \theta_{b} \\
\dot{y}_{b}=\dot{X}_{b} \sin \theta_{b}+\dot{Y}_{b} \cos \theta_{b} \\
\dot{z}_{b}=\dot{Z}_{b}
\end{array}\right.
$$

with:

$$
\left(\begin{array}{c}
\dot{X}_{b} \\
\dot{Y}_{b} \\
\dot{Z}_{b}
\end{array}\right)=\frac{\Omega R}{1-e \cos \Psi_{b}}\left(\begin{array}{c}
-\cos \mu \\
\sin \Psi_{b} \\
\sqrt{1-e^{2}} \cos \Psi_{b} \\
\sin \mu \sin \Psi_{b}
\end{array}\right)
$$

Once the positions and velocities are known, it is easy to implement the time delays up to order 1. Fig 123] show the contributions of orders $0,1 / 2$ and 1 respectively. We can also evaluate the Sagnac term as the difference between the propagation time from spacecraft 2 to 3 and the time from 3 to 2: see Fig 4 Remark that the maximum amplitude of this differential time delay is approximately twice the maximum amplitude of the ${ }^{(1 / 2)}$ contribution, because there is a change of sign. Annual variations of the frequency shift at order $1 / 2$ are represented on Fig [5] The contribution of order 1 is shown on Fig 6

\section{CONCLUSION}

We have given the formulas that allow one to compute the time delays to be used in a numerical model of LISA knowing the Keplerian orbits of the spacecraft. At the lowest order, we have the well-known "flexing" of the triangle, of (peak to peak) amplitude slightly less than $48,000 \mathrm{~km}$. At the next order, we find an extra correction of amplitude about $960 \mathrm{~km}$, larger than the allowed error for phase noise cancellation after TDI. The last computed 
order is negligible (less than $30 \mathrm{~m}$ ). The interest of the derivation is that it includes naturally all relativistic effects (for instance the Sagnac effect) introduced up to now by "ad hoc" considerations.

We have also estimated the global frequency shift due to the various motions and to propagation in the gravitational field of the Sun. We have found the rather surprising result that the General Relativistic contribution is much smaller than a priori expectations, only due to the LISA configuration. The global frequency shift, reduced to the residual relative motions of the spacecraft, is of the order of $8 \mathrm{MHz}$ (peak to peak).

\section{ACKNOWLEDGEMENT}

S. Pireaux acknowledges a CNES postdoctoral grant and a convention CNES-INSU 02/CNES/282. 


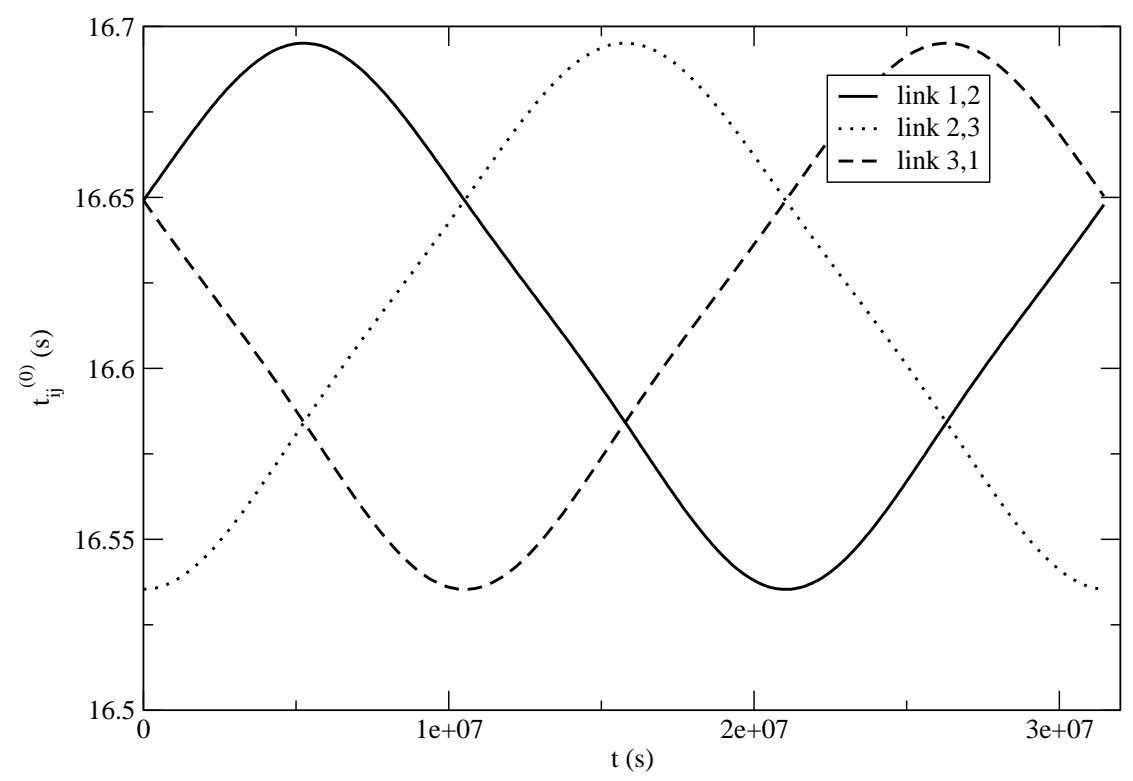

FIG. 1: Annual variations of the time delays (zeroth order) along the LISA arms. Solid line: $t_{12}^{(0)}$, short dashed line: $t_{23}^{(0)}$, long dashed line: $t_{31}$.

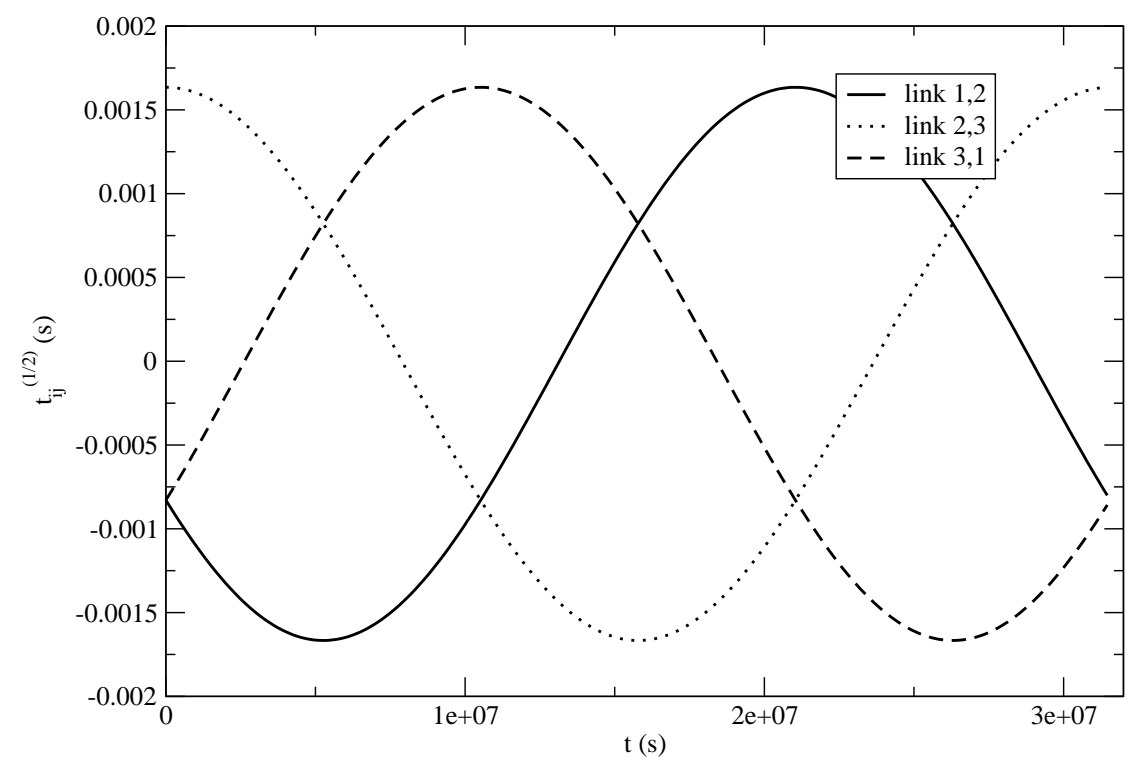

FIG. 2: Annual variations of the contribution of order $1 / 2$ to the time delays. Solid line: $\stackrel{(1 / 2)}{t_{12}}$, short dashed line: $t_{23}$, long dashed line: ${ }^{(1 / 2)}$ 


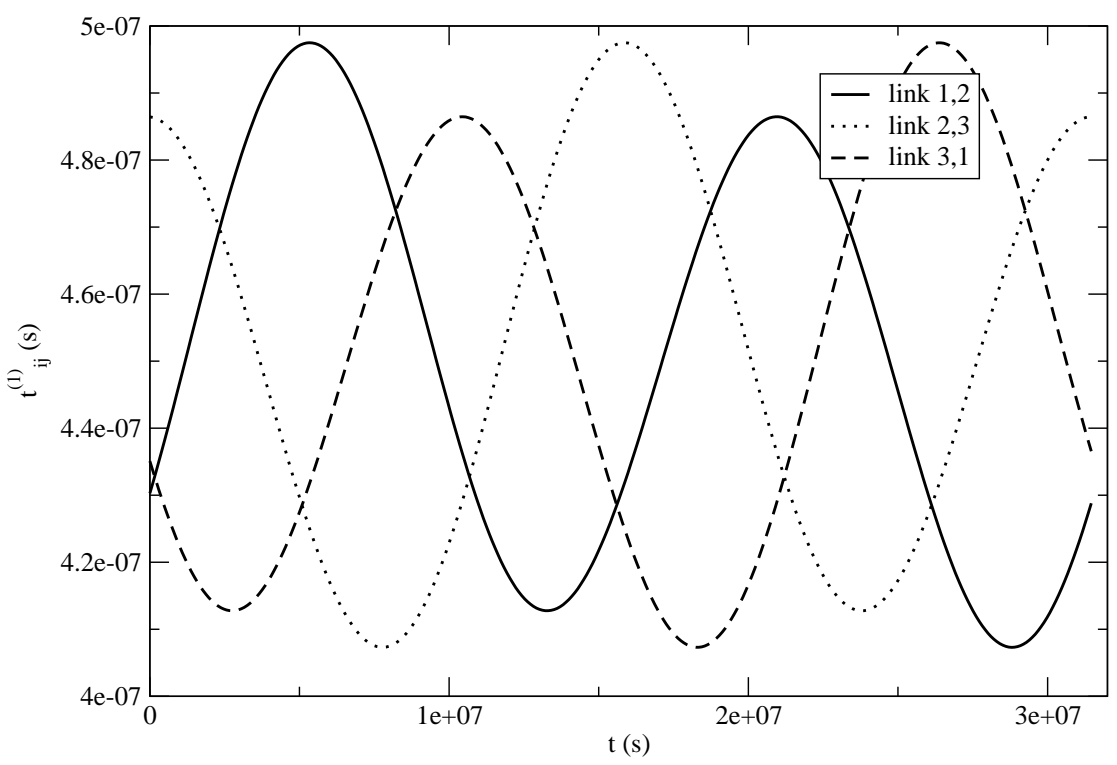

FIG. 3: Annual variations of the contribution of order 1 to the time delays. Solid line: $t_{12}^{(1)}$, short dashed line: $t_{23}$, long dashed line: $t_{31}$.

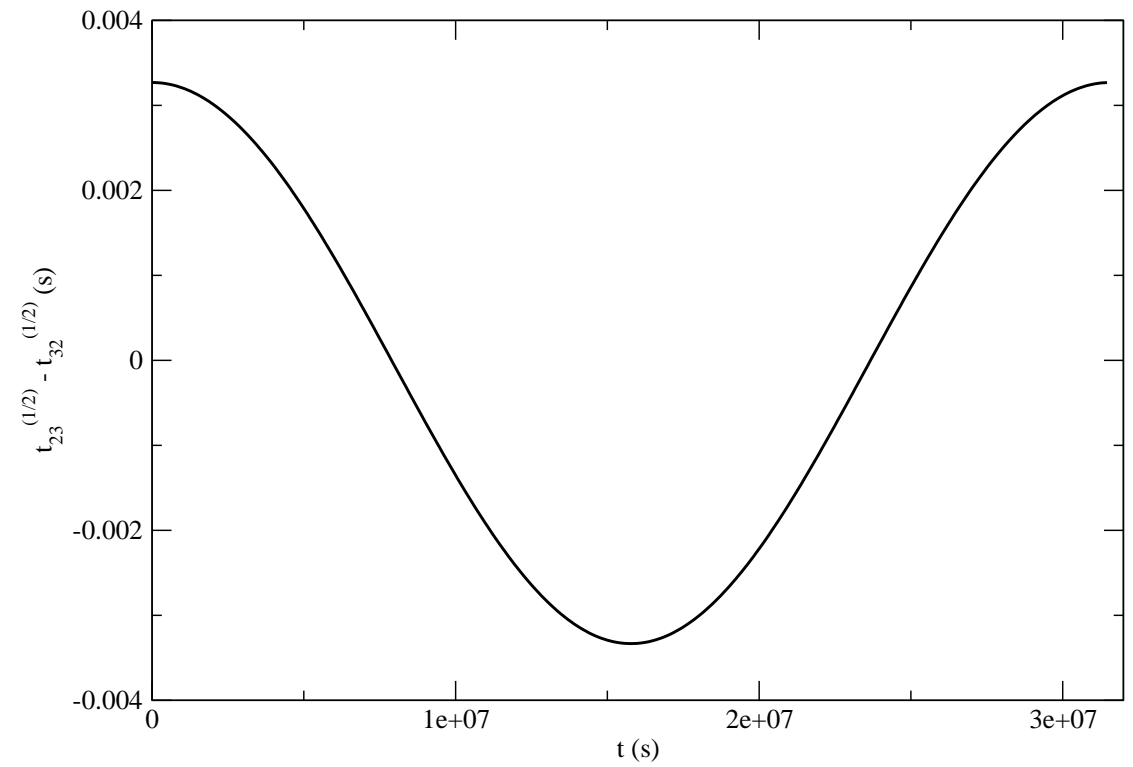

FIG. 4: Annual variations of the Sagnac differential time between spacecraft 2 and spacecraft 3. 


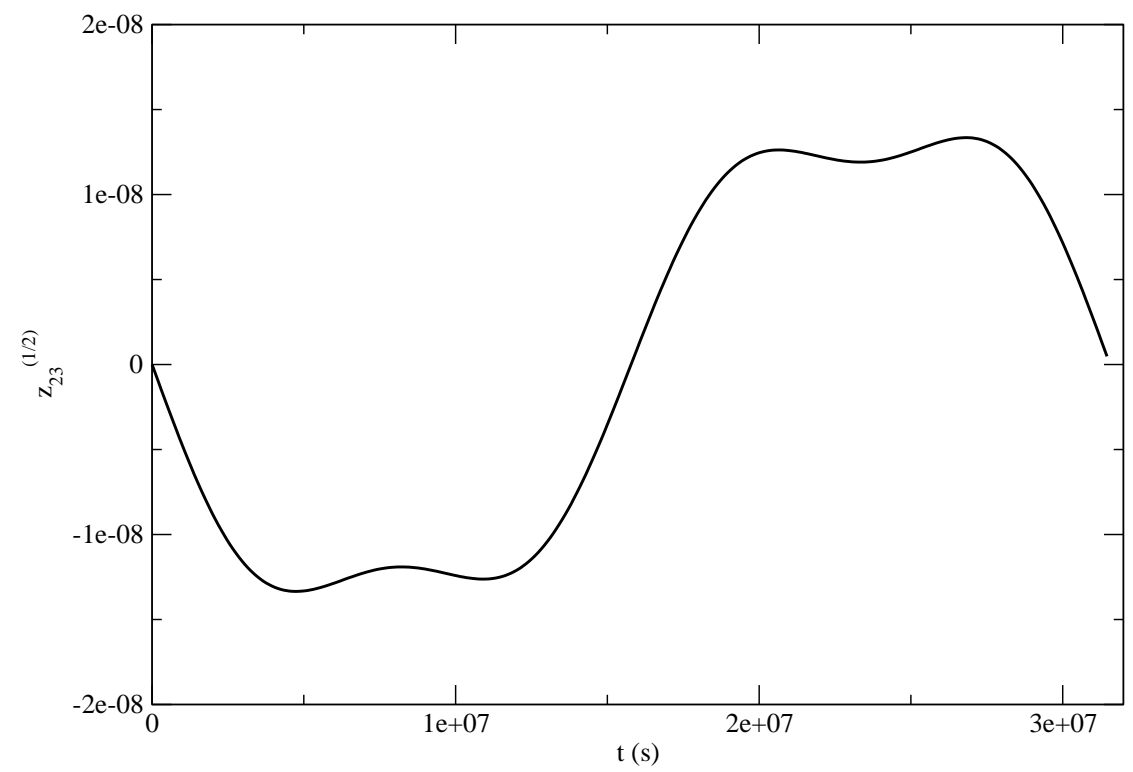

FIG. 5: Annual variations of the contribution of order $1 / 2$ to the frequency shift.

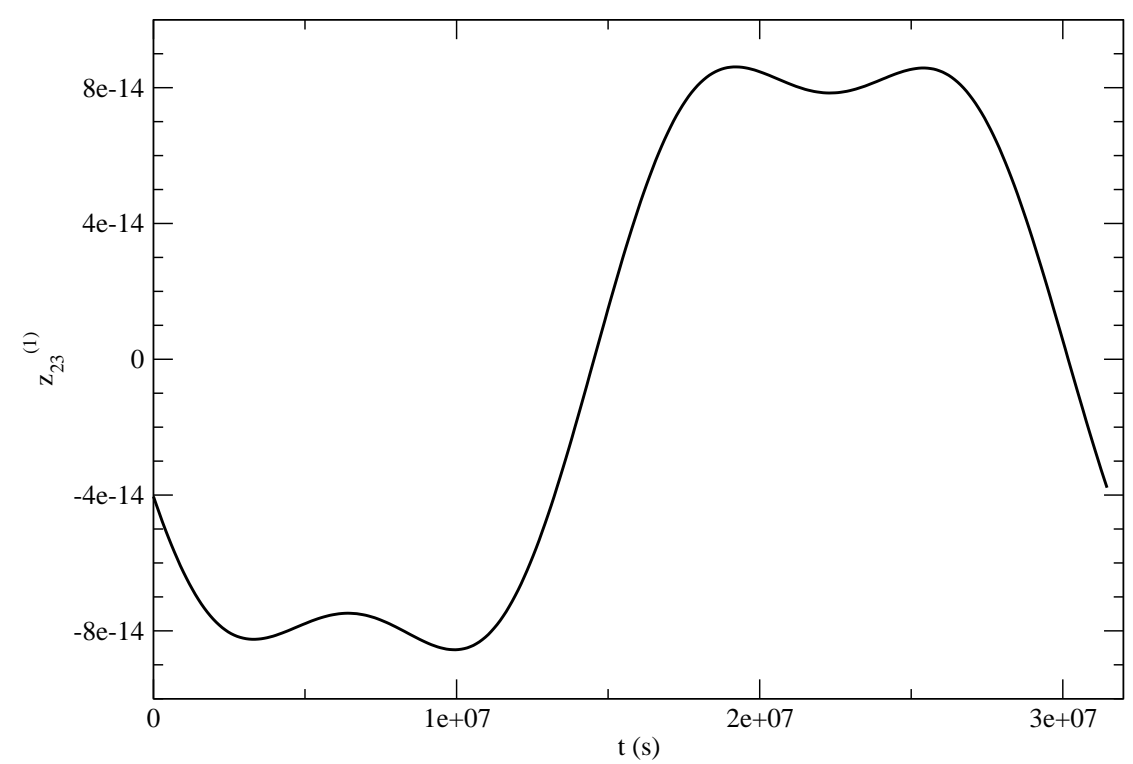

FIG. 6: Annual variations of the contribution of order 1 to the frequency shift. 
[1] S. Dhurandhar, K.R. Nayak and J.-Y. Vinet, Classical and Quantum Gravity 22 (2005) p.481

[2] J.W. Armstrong, F.B. Estabrook and M. Tinto, Phys. Rev. D 62, 042002 (2000)

[3] S.V. Dhurandhar,K.R. Nayak and J-Y. Vinet, Phys. Rev. D 65, 102002 (2002)

[4] H.S. Ruse,

Proc. London Math. Soc., 31, 225 (1930);

Proc. London Math. Soc., 32, 87 (1931)

[5] J.L. Synge,

Proc. London Math. Soc., 32,241 (1931);

Relativity: the General Theory (Amsterdam: NorthHolland) 1964
[6] B. Linet and P. Teyssandier, Phys. Rev. D 66, 024045 (2002)

[7] L. Blanchet, C. Salomon, P. Teyssandier and P. Wolf, A\&A 370, 320-329 (2001)

[8] C. Le Poncin-Lafitte, B. Linet and P. Teyssandier, Classical and Quantum Gravity 21 p.4463 (2004)

[9] N.J. Cornish and L.J. Rubbo, Phys. Rev. D 67022001 (2003)

[10] M. Vallisneri gr-qc/0407102

[11] K.R. Nayak, S. Koshti, S.V. Dhurandhar and J.-Y.Vinet submitted 